\title{
Comprehensive Study on the Potential Therapeutic Use of Brevetoxin-2 to Aid Post-stroke Recovery
}

\author{
Erica Sequeira, Marsha Pierce, Dina Gomez, Thomas Murray \\ Pharmacology, Creighton University, USA
}

OBJECTIVE Stroke triggers changes in neuronal circuits in the peri-infarct area which plays a key role in recovery and represents a potential therapeutic target. Brevetoxin-2 ( $\mathrm{PbTx}-2)$ interacts with voltage gated sodium channels. Previous studies found that $\mathrm{PbTx}-2$ influences the activation of key signaling proteins that alter physiologic events involved in synaptic plasticity and survival of neurons.

HYPOTHESES PbTx-2 will aid in functional recovery post-stroke by increasing dendritic arbor complexity and excitatory synapse density in the peri-infarct region

METHODS A photothrombotic stroke was produced by administration of an intraperitoneal injection of Rose Bengal followed by a 12.5 min light illumination of the motor cortex region resulting in clotting in YFP mice. Epicortical application of $\mathrm{PbTx}-2$ was applied over the infarct area on day 5 post-stroke. Pasta matrix reach task (PMRT) was used to assess the ability of mice to grasp pasta pieces. A second motor test, the foot fault test (FFT), was performed where the mouse could freely walk on an elevated wire grid and assessed for foot faults. Brain sections were imaged using a Leica confocal microscope and analyzed with Imaris software to measure dendritic arbor complexity. Immunohistochemistry (IHC) was performed using VGlut1 and PSD95 as synaptic markers to measure synapse density that was analyzed by Volocity Software.

RESULTS The number of pasta pieces retrieved was significantly impaired on day 1 post-stroke in all animals with stroke. There was subsequent improvement of this task following treatment with PbTx-2 as compared to the controls on day 6. In addition, the PbTx-2 treated mice had a decrease in the number of foot faults in comparison to the controls on day 6 . $\mathrm{PbTx}-2$ treated layer $\mathrm{V}$ pyramidal neurons had a two-fold increase in dendritic arbor complexity as compared to controls. Preliminary IHC data suggest that the synapse density in the peri-infarct region was increased in $\mathrm{PbTx}-2$ treated mice compared to the control mice.

CONCLUSION: Preclinical research in a murine stroke model provided behavioral assessments of neural insult-induced functional deficits and $\mathrm{PbTx}-2$ induced motor recovery. Increased dendritic arbor complexity and excitatory synapse density indicates that $\mathrm{PbTx}-2$ can potentially augment neuroplasticity in the peri-infarct region. 